\title{
The Importance of the Pulmonic Valve Morphology in Pulmonary Hypertension
}

\author{
Timothy E Paterick* \\ Aurora Bay Care Clinic, USA
}

*Corresponding author: Timothy E Paterick, Aurora Health Care, Green Bay/ Milwaukee, WI, USA.
Received Date: July 25, 2019

Published Date: July 31, 2019

\begin{abstract}
Pulmonic valve prolapse has been described in association with the mxyomatous "floppy" mitral valve syndrome and in congenital heart disease associated with pulmonary hypertension. A literature search suggests this finding of an isolated mxyomatous pulmonary valve with prolapse in adults with pulmonary hypertension is primarily or due to left sided heart conditions. This case report describes an isolated mxyomatous "floppy" pulmonic valve in a patient with primary pulmonary hypertension. Prolapse of the pulmonic valve is an echocardiographic qualitative hint of pulmonary hypertension. It is plausible to explain the morphological changes of the pulmonary valve as due to exaggeration of pulmonic valve excursion due to severely elevated pulmonary artery diastolic pressure..
\end{abstract}

Keywords: Pulmonic valve morphology; Pulmonic valve prolapse; Myxomatous pulmonic valve; Pulmonary hypertension

Learning Objectives

1. Recognition of the association between pulmonary valve prolapse and pulmonary hypertension

2. The importance of careful and dedicated imaging of the pulmonary valve.

3. Recognize the sensitivity, specificity and positive predictive value of isolated mxyomatous pulmonic valve in patients with pulmonary hypertension have not been established but the association is real.

\section{Case Report}

A 40-year-old male was admitted to the hospital with pleuritic chest pain and progressively increasing shortness of breath. He had been experiencing racing heart sensation for the previous four weeks. Past medical history was significant for hypertension and elevated body mass index. He denied pre-syncope or loss of consciousness. Physical examination revealed an obese male (BSA 2.4), with a blood pressure of $180 / 100$ and pulse of 98 bpm. Heart tones were distant without ejection click or systolic or diastolic heart murmur. Crackles were appreciated in lower lung fields. There was pitting edema in the lower extremities.

The chest X-ray revealed small bilateral pleural effusion and enlarged pulmonary arteries. Urgent chest commuted tomography revealed no evidence of pulmonary emboli but commented on enlarged pulmonary arteries.

Echocardiography revealed unusual pulmonary valve morphology with a myxomatous pulmonary valve with pulmonic valve prolapse, (Movie 1-3) and moderate pulmonary regurgitation. (Movie 4) Echo Doppler interrogation revealed elevated pulmonary regurgitation end-diastolic velocity suggesting elevated pulmonary artery end diastolic pressure. The right ventricle was remodeled with severe enlargement and depressed right ventricular function. The inferior vena cava and hepatic veins were plethoric, (Movies $5,6)$ and the tricuspid regurgitation jet revealed severe pulmonary hypertension (Movie Still 7). Right heart cardiac catheterization revealed severe elevation of pulmonary artery diastolic and systolic pressure concordant with the echocardiographic findings. Blood cultures were negative. CBC, CHEM-7, sedimentation rate and urinalysis were normal. The pulmonary hypertension team was consulted.

\section{Discussion}

Isolated pulmonary valve prolapse may be associated with pulmonary hypertension [1]. Prolapse of the pulmonic valve 
represents exaggerated anterior displacement of the pulmonary valve from the normal pulmonary valve plane [1]. The reason for pulmonary valve prolapse is enigmatic. The normal pulmonary valve is pliable and therefore reactive to slight changes in pressure. Pliability is maintained because the valve is exposed to low pressures under normal circumstances. A high pulmonary pressure may inevitably exaggerate the normal backward convex motion of the elastic pulmonary valve leaflets to result in pulmonary valve prolapse on the two-dimensional echocardiogram.

Pulmonary valve prolapse is a relatively specific valvular abnormality in patients with pulmonary hypertension [1]. Pulmonary valve prolapse is the only specific valvular abnormality that has been identified in patients with pulmonary hypertension. An intense echocardiographic signal of a morphologically thick pulmonary valve secondary to congenital left-to-right shunts has been identified as an ultrasound marker for pulmonary hypertension [2, 3]. However, a dense pulmonary valve may also be seen in carcinoid syndrome, bacterial endocarditis, and congenital pulmonary stenosis. This case is unique because a literature search does not identify a prolapsing mxyomatous pulmonary valve in normal individuals or pathological conditions that affect the pulmonary valve other than in mxyomatous "floppy" mitral valve prolapse syndrome.

The sensitivity, specificity and positive predictive value of isolated mxyomatous pulmonic valve in patients with pulmonary hypertension have not been established. The evolution of the ultrasound imaging in conjunction with right heart catheterization should allow us to address this association. In this case both echocardiographic Doppler interrogation and right heart catheterization hemodynamics reveal severe pulmonary hypertension. This association between pulmonic valve prolapse and pulmonary hypertension is extremely important because often we are unable to obtain a tricuspid envelope that determines the gradient between the right ventricle and right atrium. Pulmonic valve prolapse is a useful noninvasive imaging signal for detecting pulmonary hypertension. This case reinforces the importance of detailed assessment of pulmonic valve morphology and hemodynamics when assessing patients for pulmonary hypertension.

\section{Conclusion}

The association between pulmonic valve prolapse and pulmonary hypertension is extremely important because often we are unable to obtain a tricuspid envelope that determines the gradient between the right ventricle and right atrium. Pulmonic valve prolapse is a useful noninvasive imaging signal for detecting pulmonary hypertension. The evolution of the ultrasound imaging in conjunction with right heart catheterization should allow us to address this association between pulmonary valve prolapse and pulmonary hypertension

Movie 1: Short axis view of the pulmonic valve revealing mxyomatous valve cusps

Movie 2: Long -axis of the pulmonic valve demonstrating: prolapse of the pulmonic valve leaflets.

Movie 3: Long axis of the pulmonic valve demonstrating: the mxyomatous anterior and posterior leaflets.

Movie 4: Long axis of the pulmonary valve with color flow: Doppler revealing moderate pulmonary valve regurgitation.

Movie 5: Modified apical 4 chamber revealing: the right ventricle is remodeled with enlargement, depressed RV systolic function and flat septum.

Movie 6: Plethoric IVC without respiratory variation suggesting elevated right atrial pressure.

Movie 7: Tricuspid Valve regurgitation demonstrating RV - RA $=100 \mathrm{~mm} \mathrm{Hg}$

\section{Acknowledgement}

None.

\section{Conflict of Interest}

No conflict of interest.

\section{References}

1. Weyman AE, Dillon JC, Feigenbaum H, Chang S (1974) Echocardiographic patterns of pulmonic valve motion with pulmonary hypertension. Circulation 50(5): 905-910.

2. Peterj Robinson, Richard KH Wyse, Fergus Macartne (1984) Significance of pulmonary valve prolapse A cross sectional echocardiographic study. Br Heart J 52(3): 266-271.

3. Haneda T, Niwa T, Miura T, Miyazawa K, Takishima T, et al. (1981) Echocardiographic and morphological features of the pulmonic valve in patients with primary pulmonary hypertension and those with secondary pulmonary hypertension resulting from left-to-right shunt. Tohoku J Exp Med. 1981 August 134(4): 393-400. 